Benedikt George ${ }^{\star}$, Michael Fink, Stefan Lyer, Christoph Alexiou, Helmut Ermert, and

Stefan J. Rupitsch

\title{
Guiding and Accumulation of Magnetic Nanoparticles Employing High Intensity Focused Ultrasound for Drug Targeting Applications
}

https://doi.org/10.1515/cdbme-2019-0148

\begin{abstract}
Magnetic Drug Targeting (MDT) is a cancer treatment technique that enables a local chemotherapy. In MDT, chemotherapeutic drugs are bound to magnetic nanoparticles and are accumulated in the tumor area by means of an external magnetic field. Unfortunately, a single magnet can only generate a pulling magnetic force. However, in some applications a pushing force on the nanoparticles could be advantageous. One way to realize pushing forces is to exploit the acoustic radiation force based on the nonlinearity of sound propagation in fluid media generated by a high intensity focused ultrasonic transducer. In this context, we built a test setup was built to investigate the utility of High Intensity Focused Ultrasound (HIFU) to generate a pushing force on the magnetic nanoparticles. The results show that the acoustic radiation force can be employed for particle guidance to achieve concentration differences similar to those obtained by using an electromagnet.
\end{abstract}

Keywords: Ultrasound, acoustic radiation force, nanoparticles, drug targeting.

\section{Introduction}

A reduction of overall medication dosage to minimize side effects for cancer patients is still a major issue in cancer therapy. Therefore, various treatment methods are developed and investigated in laboratory tests concerning their efficacy and feasibility [1], [2]. Recent investigations have shown that MDT is a suitable method for an improved local chemotherapy, as the total medication dosage can be reduced [3]. In comparison to conventional chemotherapy where large drug quantities are injected into the body, the drug is bound to magnetic

*Corresponding author: Benedikt George, Department of Sensor Technology, Paul-Gordan-Street 3/5, Erlangen, Germany, e-mail: benedikt.george@fau.de

Michael Fink, Helmut Ermert, Stefan J. Rupitsch, Department of Sensor Technology, Paul-Gordan-Street 3/5, Erlangen,

Germany

Stefan Lyer, Christoph Alexiou, Section of Experimental Oncology and Nanomedicine, Glückstraße 10a, Erlangen, Germany nanoparticles in MDT and less of the toxic pharmaceutical is required. These nanoparticles are used as drug carriers and are accumulated by means of an external magnetic field in the tumorous tissue, whereby the total administered dosage can be reduced and at the same time, an increased concentration of chemotherapeutic agent in the tumor area can be achieved [4]. In conventional chemotherapy, the active component is administered in various ways (e.g. per-orally, intravenously, subcutaneously, intramuscular), whereas in MDT the nanoparticles are applied intra-arterially close to the tumor, enabling highly concentrated chemotherapy in the vessels leading to the tumor. Accumulating the magnetic particles inside the tumor requires a force from outside the body. For this purpose, MDT applies a strong electromagnet generating a magnetic force to attract the particles and pull them to the targeted region. However, the magnetic force is only effective as a pulling force but for some applications, a pushing force could be advantageous. Moreover, the magnetic field is rapidly decaying outside the magnet and inside the body, limiting medical application to nearsurface tumors. This indicates the need for the investigation of an additional treatment method to accumulate the nanoparticles not only from a different force direction but also in different tissue depths. The acoustic radiation force, induced by a HIFU transducer, could be a conceivable method to navigate nanoparticles through the bloodstream system and to enrich the nanoparticles in the tumor tissue. One has to mention that the acoustic radiation force respectively radiation pressure is a non-linear effect occuring by the propagation of high intensity ultrasound in quasi-fluid media like soft tissue. Due to the consisting harmonics and sub-harmonics including zero frequency spectral components, a constant pressure, the radiation pressure, is formed in addition to the alternating sound pressure. The effect of the radiation pressure on a surface, e.g., the surface of a particle in the sound field, leads to a force acting on the particle. The temporal mean acoustic radiation force $\bar{F}$ on a small spherical, incompressible particle with dimensions that are smaller than the acoustic wavelength $\lambda$, is calculated 
as follows [5], [6]

$$
\bar{F}=4 \pi R^{2} \frac{\bar{p}^{2}}{\varrho_{0} c_{0}^{2}}(k R)^{4}\left[1+\frac{(1-a)^{2}}{\left(2+a^{2}\right)}\right] .
$$

In (1), $\bar{F}$ is given by the radius $R$ of the nanoparticle, the temporal mean acoustic pressure $\bar{p}$, the density $\varrho_{0}$ of the sound propagation medium, the acoustic velocity $c_{0}$, the wave number $k$, and the ratio $a=\varrho_{0} / \varrho_{p}$ of the density of the sound propagation medium $\varrho_{0}$ and the particle density $\varrho_{p}$. As the density of iron oxide is higher than the density of blood, we can assume that the acoustic radiation force can be utilized to guide the particles, even though their diameter is in the nanometer range.

In order to investigate whether the acoustic radiation force can be applied to guide drug carrying nanoparticles through the vascular system, a test bench was built that emulates the anatomy of the vascular system.

\section{Experimental Setup}

The experimental setup is limited to the essential components of the blood circuit containing the human heart, the aorta and the vascular system. The human heart is represented by a peristaltic pump, a damping element (air filled hose) is acting as the aorta, and a pipeline that branches into two sub-pipes is acting as a vessel bifurcation, which is reproduced inside a tissue-mimicking phantom (Fig. 1(1)).
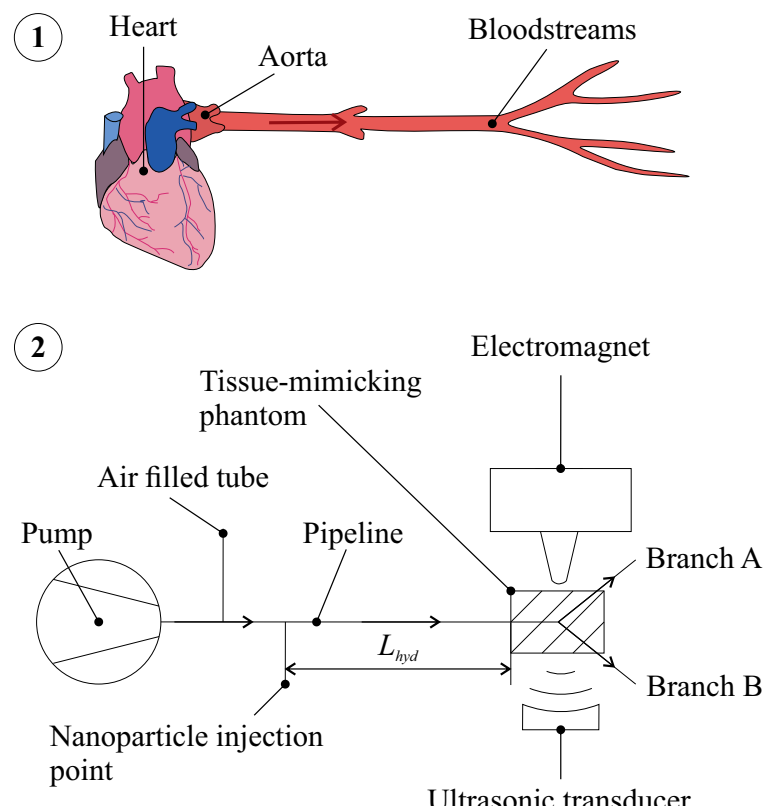

Fig. 1: Simplified hydraulic setup of the human cardiovascular system (1) compared to the experimental setup (2).
In order to realize laminar flow conditions - as it is mostly the case in the human body [7] - within the pipeline system, it is necessary to be able to adjust the displacement volume of the pump. Hence, a $24 \mathrm{~V}$ DC powered peristaltic pump (Boxer Pumps 15KS) - supplied by a DC power unit (Voltcraft DPS-4005PFC) - with settable flowrates ranging from 0 to $580 \mathrm{ml} / \mathrm{min}$ is used. Typical for a positive displacement pump is a pulsating flow, which accentuates the need for a damping object that is implemented via the air-filled tube working as an air vessel and concurrently emulates the aorta. Preventing turbulent flow inside the conduit and inside the bifurcation leads to a pump rotation speed limit of $300 \mathrm{rpm}$, which corresponds to a volume flow of $219 \mathrm{ml} / \mathrm{min}$.

With respect to the cardiovascular system, the inner diameter of the pipeline is $3 \mathrm{~mm}$ [8]. As laminar flow conditions are needed, a hydraulic entrance length $L_{h y d}=400 \mathrm{~mm}$ (Fig. 1(2)) is calculated with a critical Reynolds number $R e$ equal to 2300 and the tube diameter $d=3 \mathrm{~mm}$ [9]. In addition, pure water, which is a Newtonian fluid, is utilized as blood mimicking fluid in which the nanoparticles are injected. By using water, unsteady flow properties that emerge in nonNewtonian fluids (e.g., changing viscosity) can be avoided. The nanoparticles are injected via a syringe driver (Braun Perfusor fm), which is usually employed for long-term accurate medication in hospitals, enabling programmable volumetric dosing ranging from $0.1 \mathrm{ml} / \mathrm{h}$ up to $200 \mathrm{ml} / \mathrm{h}$.

The nanoparticles used in this work consist of consist of $20 \mathrm{~nm}$ diameter iron oxide cores. These are surrounded by lauric acid layers serving as linkages to the chemotherapeutic agent as well as biocompatible layers because the naked iron oxide nanoparticles exhibit intrinsic toxicity and are not stable in salt solutions or blood [10].

As described above, the bifurcation is allocated inside the tissue-mimicking phantom, consisting of polyvinyl alcohol (PVA). It has been shown that polyvinyl alcohol [11] is suitable to be used as tissue-mimicking material in medical ultrasound applications. The PVA-phantom is made of PVA granulate (DuPont Elvanol 71-30) dissolved in water and heated up to $80^{\circ} \mathrm{C}$, whereat the PVA content correlates to $10 \%$ of the phantom weight. Then, the attained material is cooled down under an bell jar to evacuate air inclusions from the material. Subsequently, the casted PVA phantom has to undergo two freeze-thaw cycles (15 hours freeze, 15 hours thaw) to obtain the essential acoustic properties of a tissue-mimicking phantom. The bifurcation is generated by recasting three $3 \mathrm{~mm}$ thick rods inside the casting mold that can be removed after the last thaw process.

The focus of the ultrasonic transducer as well as the maximum of the magnetic field gradient is coincident with the branching point of the pipeline system so that the pushing force on the nanoparticles should increase the particle concen- 
tration in branch A of the pipeline system. The HIFU transducer and the tissue-mimicking phantom are positioned in a water tank.

\section{Measurement Results}

The ultrasonic transducer emitted a focused ultrasonic wave at $835 \mathrm{kHz}$ and the pressure value in the focus point was $0.63 \mathrm{MPa}$, inducing a mechanical index of $\mathrm{MI}=0.69$, which is below the maximum value that the American Institute of Ultrasound in Medicine mandates for sonication in diagnostic applications. The electromagnet was not operated during the ultrasonic test series. Furthermore, the pumps revolution speed was chosen to be $100 \mathrm{rpm}$ leading to a flow rate of $73 \mathrm{ml} / \mathrm{min}$ and the syringe output was set to $66.7 \mathrm{ml} / \mathrm{h}$. To verify the change of particle concentration, the content of iron in both branches was determined at the Section of Experimental Oncology and Nanomedicine (SEON, University-Hospital Erlangen) using microwave plasma atomic emission spectrometry. After the measurements, the particle concentration in each of the branches was determined.

Before reaching the branching point, the iron content in the liquid was approximately $2920 \mu \mathrm{g} / \mathrm{l}$. After sonication (Fig. 2(1)), the iron content in branch A increased to a mean value of $4380 \mu \mathrm{g} / \mathrm{l}$ and decreased in branch B to a mean value of $2470 \mu \mathrm{g} / \mathrm{l}$, resulting in an absolute concentration difference of $1910 \mu \mathrm{g} / \mathrm{l}$. Compared to the accumulation with the electromagnet (Fig. 2(2)), one can determine this iron concentration difference nearly at a current of almost $52.5 \mathrm{~A}$. This indicates a strong magnetic field gradient and therefore suggests an efficient ultrasonic accumulation.

\section{Conclusion}

Without acoustic irradiation of the branching point, the nanoparticles spread evenly over the two branches with equal particle densities. With acoustic irradiation, the nanoparticle flow could be controlled in such a way that the content of iron increased significantly in branch A as opposed to branch B. This proved that the acoustic radiation force could be employed for particle guiding and accumulation in drug targeting applications.
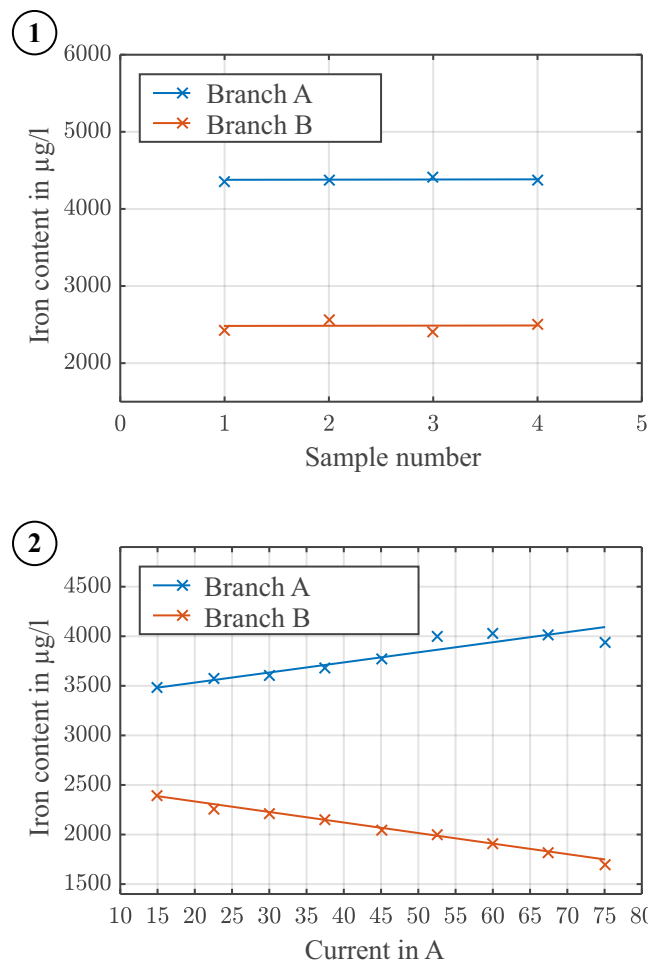

Fig. 2: Results of the particle concentration gained with US (1) compared to the accumulation with the electromagnet (2).

\section{References}

[1] Torchilin VP. Drug targeting. European Journal of Pharmaceutical Sciences 2000;11:81-91.

[2] Tietze R, Lyer S, Dürr S, Struffert T, Engelhorn T, Schwarz M, et al. Efficient drug-delivery using magnetic nanoparticlesbiodistribution and therapeutic effects in tumour bearing rabbits, Nanomed. 2013;9:961-971.

[3] Alexiou C, Schmid R. J, Jurgons R, Kremer M, Wanner G, Bergemann C, et al. Targeting cancer cells: Magnetic nanoparticles as drug carriers. European Biophysics Journal 2006;35:446-450.

[4] Alexiou C, Schmidt A. J, Klein R, Hulin P, Bergemann C, Arnold W. Magnetic Drug Targeting: Biodistribution and dependency on magnetic field strength. Journ. Magnetism Magn. Mater. 2002;252:446-450.

[5] Sutilov V. A, Fundamental physics of ultrasound. Gordon and Breach Science Publisher, 1988.

[6] King L. V, On the acoustic radiation pressure on spheres. Bd. A 147. Proc. Roy. Soc., 212-240, 1934.

[7] Herman IP. Physics of the Human Body. Berlin: Springer; 2005.

[8] Schaal S, Kunsch K, Kunsch S. Der Mensch in Zahlen. Berlin Heidelberg: Springer Spektrum; 2016.

[9] Herwig H. Strömungsmechanik. Wiesbaden: Springer Vieweg; 2016.

[10] J. Zaloga, C. Janko, J. Nowak, J. Matuszak, S. Knaup, D. Eberbeck, et al. Development of a lauric acid/album hybrid 
iron oxide nanoparticle system with improved biocompatibility. Intern. Journ. Nanomed. 2014;9 4847-4866.

[11] Surry KJM, Austin HJB, Fenster A, Peters TM. Poly (vinyl alcohol) cryogel phantoms for use in ultrasound and MR imaging. Phys. in Med. and Biol. 49, 5529-5546, 2004. 